\title{
On the generators of a generalized numerical semigroup
}

\author{
Carmelo Cisto, Gioia Failla and Rosanna Utano
}

\begin{abstract}
We give a characterization on the sets $A \subseteq \mathbb{N}^{d}$ such that the monoid generated by $A$ is a generalized numerical semigroup (GNS) in $\mathbb{N}^{d}$. Furthermore we give a procedure to compute the hole set $\mathbb{N}^{d} \backslash S$, where $S$ is a GNS, if a finite set of generators of $S$ is known.
\end{abstract}

\section{Introduction}

Let $\mathbb{N}$ be the set of non negative integers. A numerical semigroup is a submonoid $S$ of $\mathbb{N}$ such that $\mathbb{N} \backslash S$ is a finite set. The elements of $H(S)=\mathbb{N} \backslash S$ are called the holes of $S$ (or gaps) and the largest element in $H(S)$ is known as the Frobenius number of $S$, denoted by $F(S)$. The number $g=|H(S)|$ is named the genus of $S$. It has been proved that every numerical semigroup $S$ has a unique minimal set of generators $G(S)$, that is in $S$ every element is a linear combination of elements in $G(S)$ with coefficients in $\mathbb{N}$. Furthermore the set of minimal generators of a numerical semigroup is characterized by the following: the set $\left\{a_{1}, a_{2}, \ldots, a_{n}\right\}$ generates a numerical semigroup if and only if the greatest common divisor of the elements $a_{1}, a_{2}, \ldots, a_{n}$ is 1 . For the background on this subject, a very good reference is [9].

In [3] it is provided a straightforward generalization of numerical semigroups in $\mathbb{N}$ for submonoids of $\mathbb{N}^{d}$ : a monoid $S \subseteq \mathbb{N}^{d}$ is called a generalized numerical semigroup (GNS) if $H(S)=\mathbb{N}^{d} \backslash S$, the set of holes of $S$, is a finite set. Also

Key Words: Generalized numerical semigroup, minimal generators.

2010 Mathematics Subject Classification: Primary 20M14, 05A15; Secondary 05A16, $11 \mathrm{D} 07$.

Received: 27.01 .2018

Accepted: 27.04 .2018 
in this case the cardinality of $\mathbb{N}^{d} \backslash S$ is called the genus of $S$. In [3] the tree of generalized numerical semigroups is efficiently calculated up to a given genus and asymptotic properties of the number of generalized numerical semigroups of a given genus are discussed. In this paper we want to extend some ideas and results for classical numerical semigroups to generalized numerical semigroups. We study basic properties of a GNS in order to characterize its minimal system of generators. More precisely, in Section 2 we prove first that every GNS in $\mathbb{N}^{d}$ has a unique minimal system of generators. Then we investigate under which conditions a finite set $A \subseteq \mathbb{N}^{d}$ generates a GNS. In Section 3, by using a connection between submonoids of $\mathbb{N}^{d}$ and power series expansions of rational functions, we deduce an algorithm to compute the set of holes of a GNS, if a finite set of generators of $S$ is given.

\section{Minimal generators}

Throughout the paper we denote by $\mathbf{e}_{1}, \mathbf{e}_{2}, \ldots, \mathbf{e}_{d}$ the standard basis vectors in $\mathbb{R}^{d}$ (that is, for $i=1, \ldots, d, \mathbf{e}_{i}$ is the vector whose $i$-th component is 1 and the other components are zero). Furthermore, if $A \subseteq \mathbb{N}^{d}$, we denote $\langle A\rangle=$ $\left\{\lambda_{1} \mathbf{a}_{1}+\cdots+\lambda_{n} \mathbf{a}_{n} \mid \lambda_{1}, \ldots, \lambda_{n} \in \mathbb{N}, \mathbf{a}_{1}, \ldots, \mathbf{a}_{n} \in A\right\}$, that is the submonoid of $\mathbb{N}^{d}$ generated by the set $A$. Moreover if $\mathbf{t} \in \mathbb{N}^{d}$, its $i$-th component is denoted by $t^{(i)}$.

Lemma 2.1. [9, Lemma 2.3] Let $S$ be a submonoid of $\mathbb{N}^{d}$. Then $S^{*} \backslash\left(S^{*}+S^{*}\right)$ is a system of generators for $S$. Moreover, every system of generators of $S$ contains $S^{*} \backslash\left(S^{*}+S^{*}\right)$.

Lemma 2.2. Let $S$ be a GNS of genus $g$ with $H(S)=\left\{\boldsymbol{h}_{1}, \boldsymbol{h}_{2}, \ldots, \boldsymbol{h}_{g-1}, \boldsymbol{h}\right\}$. Let $\boldsymbol{h}$ be a maximal element in $H(S)$ with respect to the natural partial order in $\mathbb{N}^{d}$. Then $S^{\prime}=S \cup\{\boldsymbol{h}\}$ is a $G N S$, in particular $H\left(S^{\prime}\right)=\left\{\boldsymbol{h}_{1}, \boldsymbol{h}_{2}, \ldots, \boldsymbol{h}_{g-1}\right\}$ and $S^{\prime}$ has genus $g-1$.

Proof. Let $S^{\prime}=\langle S \cup\{\mathbf{h}\}\rangle$. $S^{\prime}$ is a GNS since $S \subseteq S^{\prime}=\langle S \cup\{\mathbf{h}\}\rangle$, in particular $H(S) \supsetneq H\left(S^{\prime}\right)$. Let us prove that $S^{\prime}$ has genus $g-1$. We suppose there exists $\mathbf{h}_{j} \in H(S), j \in\{1, \ldots, g-1\}$, such that $\mathbf{h}_{j} \in S^{\prime}=\langle S \cup\{\mathbf{h}\}\rangle$. Then $\mathbf{h}_{j}=\sum_{k} \mu_{k} \mathbf{g}_{k}+\lambda \mathbf{h}$, with $\mathbf{g}_{k} \in S$. If $\lambda=0$ then $\mathbf{h}_{j} \in S$, contradiction. If $\lambda \neq 0$ then $\mathbf{h}_{j} \geq \mathbf{h}$ against the maximality of $\mathbf{h}$ in $H(S)$. So $\mathbf{h}_{j} \notin S^{\prime}$ for $j \in\{1, \ldots, g-1\}$, hence $H\left(S^{\prime}\right)=\left\{\mathbf{h}_{1}, \mathbf{h}_{2}, \ldots, \mathbf{h}_{g-1}\right\}$.

Proposition 2.3. Every GNS admits a finite system of generators.

Proof. Let $S \subseteq \mathbb{N}^{d}$ be a GNS. We prove the statement by induction on the genus $g$ of $S$. If $g=0$ then $S=\mathbb{N}^{d}$, that is generated by the standard basis vectors $\left\{\mathbf{e}_{1}, \mathbf{e}_{2}, \ldots, \mathbf{e}_{d}\right\}$. Let $S \subseteq \mathbb{N}^{d}$ be a GNS of genus $g+1$ and let $\mathbf{h}$ be a 
maximal element in $H(S)$ with respect to the natural partial order in $\mathbb{N}^{d}$. By Lemma 2.2 $S^{\prime}=S \cup\{\mathbf{h}\}$ is a GNS in $\mathbb{N}^{d}$ of genus $g$, that is finitely generated by induction hypothesis. Hence let $G\left(S^{\prime}\right)$ be a finite system of generators for $S^{\prime}$. We have $\mathbf{h} \in G\left(S^{\prime}\right)$ because $\mathbf{h}$ cannot belong to $S$. So $G\left(S^{\prime}\right) \subset S \cup\{\mathbf{h}\}$ and we can denote $G\left(S^{\prime}\right)=\left\{\mathbf{g}_{1}, \mathbf{g}_{2}, \ldots, \mathbf{g}_{s}, \mathbf{h}\right\}$ with $\mathbf{g}_{i} \in S$ for every $i=1,2, \ldots, s$. Let $\mathcal{B}=\left\{\mathbf{g}_{1}, \ldots, \mathbf{g}_{s}, \mathbf{h}+\mathbf{g}_{1}, \mathbf{h}+\mathbf{g}_{2}, \ldots, \mathbf{h}+\mathbf{g}_{s}, 2 \mathbf{h}, 3 \mathbf{h}\right\}$. By the maximality of $\mathbf{h}$ in $H(S)$ we have $\mathcal{B} \subset S$ and furthermore it is easy to prove that $\mathcal{B}$ is a system of generators for $S$. Hence $S$ is finitely generated.

Corollary 2.4. Every GNS admits a unique finite system of minimal generators.

Proof. By Lemma 2.1 every GNS admits a unique system of minimal generators, that is $S^{*} \backslash\left(S^{*}+S^{*}\right)$, which is contained in every system of generators. By Proposition 2.3 such a system of generators is finite.

Definition 2.5. Let $\mathbf{t} \in \mathbb{N}^{d}$, we define the set $\pi(\mathbf{t})=\left\{\mathbf{n} \in \mathbb{N}^{d} \mid \mathbf{n} \leq \mathbf{t}\right\}$ where $\leq$ is the natural partial order defined in $\mathbb{N}^{d}$.

Remark 2.6. Notice that for every $\mathbf{t} \in \mathbb{N}^{d}$ the set $\pi(\mathbf{t})$ is finite and it represents the set of integer points of the hyper-rectangle whose vertices are $\mathbf{t}$, its projections on the coordinate planes, the origin of axes, and the points in the coordinate axes $\left(t^{(1)}, 0, \ldots, 0\right),\left(0, t^{(2)}, 0, \ldots, 0\right), \ldots,\left(0, \ldots, 0, t^{(d)}\right)$. If $\mathbf{s} \notin \pi(\mathbf{t})$ then $\mathbf{s}$ has at least one component larger than the respective of $\mathbf{t}$.

Lemma 2.7. Let $S \subseteq \mathbb{N}^{d}$ be a monoid. Then $S$ is a GNS if and only if there exists $\boldsymbol{t} \in \mathbb{N}^{d}$ such that for all elements $\boldsymbol{s} \notin \pi(\boldsymbol{t})$ then $\boldsymbol{s} \in S$.

Proof. Let $S$ be a GNS in $\mathbb{N}^{d}$ whose hole set is $H(S)=\left\{\mathbf{h}_{1}, \mathbf{h}_{2}, \ldots, \mathbf{h}_{g}\right\}$. Let $t^{(i)} \in \mathbb{N}$ be the largest number appearing in the $i$-th coordinate of elements in $H(S)$ for $i \in\{1, \ldots, d\}$, in other words $t^{(i)}=\max \left\{h_{1}^{(i)}, h_{2}^{(i)}, \ldots, h_{g}^{(i)}\right\}$. It is easy to see that $\mathbf{t}=\left(t^{(1)}, t^{(2)}, \ldots, t^{(d)}\right) \in \mathbb{N}^{d}$ fulfils the thesis.

Conversely, let $\mathbf{t} \in \mathbb{N}^{d}$ be an element such that for every $\mathbf{s} \notin \pi(\mathbf{t})$ it is $\mathbf{s} \in S$. Therefore if $\mathbf{h} \in \mathbb{N}^{d} \backslash S$ then $\mathbf{h} \in \pi(\mathbf{t})$, that is $\left(\mathbb{N}^{d} \backslash S\right) \subseteq \pi(\mathbf{t})$ and since $\pi(\mathbf{t})$ is a finite set then $S$ is a GNS.

For the proof of the next theorem, that is the main result of this paper, we consider that the Frobenius Number of $\mathbb{N}$ (the trivial numerical semigroup) is 0 , although it is usually defined to be -1 in the existing literature.

Theorem 2.8. Let $d \geq 2$ and let $S=\langle A\rangle$ be the monoid generated by a set $A \subseteq \mathbb{N}^{d}$. Then $S$ is a GNS if and only if the set $A$ fulfils each one of the following conditions: 
1. For all $j=1,2, \ldots, d$ there exist $a_{1}^{(j)} \boldsymbol{e}_{j}, a_{2}^{(j)} \boldsymbol{e}_{j}, \ldots, a_{r_{j}}^{(j)} \boldsymbol{e}_{j} \in A, r_{j} \in$ $\mathbb{N} \backslash\{0\}$, such that $\operatorname{GCD}\left(a_{1}^{(j)}, a_{2}^{(j)}, \ldots, a_{r_{j}}^{(j)}\right)=1$ (that is, the elements $a_{i}^{(j)}, 1 \leq i \leq r_{j}$, generate a numerical semigroup).

2. For every $i, k, 1 \leq i<k \leq d$ there exist $\boldsymbol{x}_{i k}, \boldsymbol{x}_{k i} \in A$ such that $\boldsymbol{x}_{i k}=$ $\boldsymbol{e}_{i}+n_{i}^{(k)} \boldsymbol{e}_{k}$ and $\boldsymbol{x}_{k i}=\boldsymbol{e}_{k}+n_{k}^{(i)} \boldsymbol{e}_{i}$ with $n_{i}^{(k)}, n_{k}^{(i)} \in \mathbb{N}$.

Proof. $\Rightarrow$ ) If $A$ does not satisfy the first condition for some $j$ then there exist infinite elements $a \mathbf{e}_{j}, a \in \mathbb{N} \backslash\{0\}$, which do not belong to $S$. If $A$ does not satisfy the second condition for some $i \neq j$, then there are infinite elements $\mathbf{e}_{i}+n \mathbf{e}_{k}$ with $n \in \mathbb{N} \backslash\{0\}$ which do not belongs to $S$.

$\Leftarrow)$ For every $j=1,2, \ldots, d$, let $S_{j}$ be the numerical semigroup generated by $\left\{a_{1}^{(j)}, a_{2}^{(j)}, \ldots, a_{r_{j}}^{(j)}\right\}$. We denote with $F^{(j)}$ the Frobenius number of $S_{j}$. It is easy to verify that for all $n \in \mathbb{N} \backslash\{0\}$, the element $\left(F^{(j)}+n\right) \mathbf{e}_{j} \in \mathbb{N}^{d}$ belong to $S$. Let $\mathbf{v}=\left(v^{(1)}, v^{(2)}, \ldots, v^{(d)}\right) \in \mathbb{N}^{d}$ be the element defined by

$$
v^{(j)}=\sum_{\substack{i=1 \\ i \neq j}}^{d} F^{(i)} n_{i}^{(j)}+F^{(j)}
$$

for any $j=1,2, \ldots, d$. Let us prove that $\mathbf{x} \in S$ for all $\mathbf{x} \notin \pi(\mathbf{v})$ so, by Lemma $2.7, S$ is a GNS.

Let $\mathbf{x}=\left(x^{(1)}, x^{(2)}, \ldots, x^{(d)}\right) \in \mathbb{N}^{d}$ such that $x^{(j)}>v^{(j)}$ for some $j \in\{1, \ldots, d\}$. Then there exists $m_{j} \in \mathbb{N} \backslash\{0\}$ such that $x^{(j)}=v^{(j)}+m_{j}$.

If $k_{1}, k_{2}, \ldots, k_{r} \in\{1,2, \ldots, d\} \backslash\{j\}$ are such that $x^{\left(k_{i}\right)} \leq F^{\left(k_{i}\right)}$ for every $i \in\{1,2, \ldots, r\}$, so $x^{\left(k_{i}\right)} n_{k_{i}}^{(j)} \leq F^{\left(k_{i}\right)} n_{k_{i}}^{(j)}$ for every $i=1, \ldots, r$, then for every $i$ there exists $p_{i} \in \mathbb{N}$ such that $F^{\left(k_{i}\right)} n_{k_{i}}^{(j)}=x^{\left(k_{i}\right)} n_{k_{i}}^{(j)}+p_{i}$.

Moreover let $h_{1}, \ldots, h_{s} \in\{1, \ldots, d\} \backslash\{\{j\}$ be the components of $\mathbf{x}$ such that $x^{\left(h_{i}\right)}>F^{\left(h_{i}\right)}$ for every $i \in\{1, \ldots, s\}$, hence $x^{\left(h_{i}\right)} \mathbf{e}_{h_{i}} \in S$, for all $i$. 
Then we consider the following equalities:

$$
\begin{aligned}
\mathbf{x}= & \sum_{i=1}^{d} x^{(i)} \mathbf{e}_{i}=\sum_{i=1}^{r} x^{\left(k_{i}\right)} \mathbf{e}_{k_{i}}+\sum_{i=1}^{s} x^{\left(h_{i}\right)} \mathbf{e}_{h_{i}}+x^{(j)} \mathbf{e}_{j} \\
= & \sum_{i=1}^{r} x^{\left(k_{i}\right)} \mathbf{e}_{k_{i}}+\sum_{i=1}^{s} x^{\left(h_{i}\right)} \mathbf{e}_{h_{i}}+\left(\sum_{i \neq j}^{d} F^{(i)} n_{i}^{(j)}+F^{(j)}+m_{j}\right) \mathbf{e}_{j} \\
= & \sum_{i=1}^{r}\left(x^{\left(k_{i}\right)} \mathbf{e}_{k_{i}}+F^{\left(k_{i}\right)} n_{k_{i}}^{(j)} \mathbf{e}_{j}\right)+\sum_{i=1}^{s} x^{\left(h_{i}\right)} \mathbf{e}_{h_{i}}+ \\
& \left(\sum_{i=1}^{s} F^{\left(h_{i}\right)} n_{h_{i}}^{(j)}+F^{(j)}+m j\right) \mathbf{e}_{j} \\
= & \sum_{i=1}^{r}\left(x^{\left(k_{i}\right)} \mathbf{e}_{k_{i}}+\left(x^{\left(k_{i}\right)} n_{k_{i}}^{(j)}+p_{i}\right) \mathbf{e}_{j}\right)+\sum_{i=1}^{s} x^{\left(h_{i}\right)} \mathbf{e}_{h_{i}}+ \\
& \left(\sum_{i=1}^{s} F^{\left(h_{i}\right)} n_{h_{i}}^{(j)}+F^{(j)}+m_{j}\right) \mathbf{e}_{j} \\
= & \sum_{i=1}^{r} x^{\left(k_{i}\right)}\left(\mathbf{e}_{k_{i}}+n_{k_{i}}^{(j)} \mathbf{e}_{j}\right)+ \\
& \sum_{i=1}^{s} x^{\left(h_{i}\right)} \mathbf{e}_{h_{i}}+\left(\sum_{i=1}^{s} F^{\left(h_{i}\right)} n_{h_{i}}^{(j)}+\sum_{i=1}^{r} p_{i}+F^{(j)}+m_{j}\right) \mathbf{e}_{j} .
\end{aligned}
$$

Therefore $\mathbf{x}$ is a sum of elements in $S$ (note that the first sum is a linear combination of elements in $A$, whose coefficients are non negative integers). So $S$ is a GNS.

Corollary 2.9. Let $S \subseteq \mathbb{N}^{d}$ be a $G N S$ and let $A$ be a finite system of generators of $S$. With the notation of the previous theorem for the elements in $A$, let $S_{j}$ be the numerical semigroup generated by $\left\{a_{1}^{(j)}, a_{2}^{(j)}, \ldots, a_{r_{j}}^{(j)}\right\}$ and $F^{(j)}$ the Frobenius number of $S_{j}$, for $j=1, \ldots, d$. Let $\boldsymbol{v}=\left(v^{(1)}, v^{(2)}, \ldots, v^{(d)}\right) \in \mathbb{N}^{d}$ defined by:

$$
v^{(j)}=\sum_{i \neq j}^{d} F^{(i)} n_{i}^{(j)}+F^{(j)} .
$$

Then $H(S) \subseteq \pi(\boldsymbol{v})$.

Proof. It easily follows from the proof of Theorem 2.8.

Example 2.10. Let $S \subseteq \mathbb{N}^{4}$ be the GNS generated by $A=\{(1,0,0,0),(1,0,0,1)$, $(0,1,0,0),(0,1,0,1),(0,0,1,0),(0,0,2,1),(0,0,0,2),(0,0,1,3),(0,0,0,5)\}$.

Actually $S$ is a GNS and its hole set is $H(S)=\{(0,0,0,1),(0,0,0,3),(0,0,1,1)\}$. Let us verify that the conditions of Theorem 2.8 are satisfied. The generators described in condition 1$)$ of the previous theorem are $\{(1,0,0,0)$, $(0,1,0,0),(0,0,1,0),(0,0,0,2),(0,0,0,5)\}$. About the condition 2$)$ we have to verify that $A$ contains at least one element of the following shapes: 
$\left(n_{2}^{(1)}, 1,0,0\right),\left(1, n_{1}^{(2)}, 0,0\right),\left(1,0, n_{1}^{(3)}, 0\right),\left(n_{3}^{(1)}, 0,1,0\right)$ $\left(1,0,0, n_{1}^{(4)}\right),\left(n_{4}^{(1)}, 0,0,1\right),\left(0,1, n_{2}^{(3)}, 0\right),\left(0, n_{3}^{(2)}, 1,0\right)$, $\left(0,1,0, n_{2}^{(4)}\right),\left(0, n_{4}^{(2)}, 0,1\right),\left(0,0,1, n_{3}^{(4)}\right),\left(0,0, n_{4}^{(3)}, 1\right)$.

The generators described in condition 2$)$ of the previous theorem are $\{(1,0,0,0)$, $(0,1,0,0),(0,0,1,0),(1,0,0,1),(0,1,0,1),(0,0,2,1)\}$. Observe that the set $A^{\prime}=$ $A \backslash\{(0,0,1,3)\}$ is a set of generators of a GNS $S^{\prime}$, different from $S$, with a greater number of holes.

Example 2.11. Let $S \subseteq \mathbb{N}^{2}$ be the GNS whose hole set is $H(S)=\{(1,0),(2,0)$, $(2,1)\}$. The set of minimal generators of $S$ is $\{(0,1),(1,1),(3,0),(4,0),(5,0)\}$. We can identify $F^{(1)}=2, F^{(2)}=0, n_{2}^{(1)}=0, n_{1}^{(2)}=1$ so $\mathbf{v}=\left(F^{(2)} n_{2}^{(1)}+\right.$ $\left.F^{(1)}, F^{(1)} n_{1}^{(2)}+F^{(2)}\right)=(2,2)$. In Figure 1 the point $\mathbf{v}$ is marked in red, the couples of nonnegative integers in the red area represent the elements in $\pi(\mathbf{v})$.

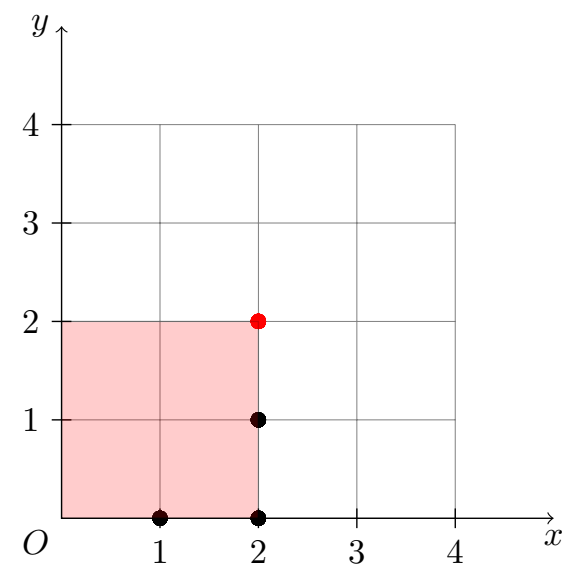

Figure 1:

The holes of $S$ are marked in black and we can see that they are all in the red area, that is $\pi(\mathbf{v})$. Moreover all the points overside the red area are in $S$. Indeed $\mathbf{v}^{\prime}=(2,1)$ satisfies Lemma 2.7 too and $\left|\pi\left(\mathbf{v}^{\prime}\right)\right|<|\pi(\mathbf{v})|$. Anyway this fact does not always occur, as we will see in the next example.

Example 2.12. Let $S \subseteq \mathbb{N}^{2}$ be the monoid generated by $G(S)=\{(2,0),(0,2)$, $(3,0),(0,3),(1,4),(4,1)\}$.

By Theorem $2.8 S$ is a GNS. Actually the hole set of $S$ is $H(S)=\{(0,1),(1,0)$, $(1,1),(1,2),(1,3),(1,5),(2,1),(3,1),(5,1)\}$. We have $F^{(1)}=1, F^{(2)}=1$, 
$n_{1}^{(2)}=4, n_{2}^{(1)}=4$, so we consider $\mathbf{v}=\left(F^{(2)} n_{2}^{(1)}+F^{(1)}, F^{(1)} n_{1}^{(2)}+F^{(2)}\right)=(5,5)$. The set $H(S)$ is contained in $\pi(\mathbf{v})$ :

In this case we can argue that it does not exist an element $\mathbf{w} \in \mathbb{N}^{2}$ such that $\pi(\mathbf{w})$ contains every hole of $S$ and $|\pi(\mathbf{w})|<|\pi(\mathbf{v})|$.

Remark 2.13. Let $S=\langle A\rangle$ be a monoid generated by $A \subseteq \mathbb{N}^{d}$. For every $j=1,2, \ldots, n$, we denote with $A_{j} \subseteq \mathbb{N}^{d-1}$ the set of the elements in $\mathbb{N}^{d-1}$, obtained from the elements in $A$ removing the $j$-th component. Then the condition 2) of Theorem 2.8 is equivalent to the following statement: for every $j=1,2, \ldots, d,\left\langle A_{j}\right\rangle=\mathbb{N}^{d-1}$.

\section{Linear combinations in $\mathbb{N}^{d}$ with coefficients in $\mathbb{N}$}

Let $S \subseteq \mathbb{N}^{d}$ be a finitely generated monoid and $\left\{\mathbf{a}_{1}, \mathbf{a}_{2}, \ldots, \mathbf{a}_{n}\right\}$ be a system of generators for $S$. We denote by $M$ the $d \times n$ matrix whose $i$-th column is the vector $\mathbf{a}_{i} \in \mathbb{N}^{d}$ for $i=1, \ldots, n$. It is easy to see that an element $\mathbf{b} \in S$ if and only if the system $M \mathbf{x}=\mathbf{b}$ admits solutions in $\mathbb{N}^{n}$. In fact this statement is equivalent to say that $\mathbf{b}$ is a linear combination of $\left\{\mathbf{a}_{1}, \mathbf{a}_{2}, \ldots, \mathbf{a}_{n}\right\} \subseteq \mathbb{N}^{d}$ with nonnegative integer coefficients.

Definition 3.1. Let $A \subseteq \mathbb{N}^{d}$ be a finite set. We define the polynomial:

$$
F_{A}=\sum_{\mathbf{v} \in A} x^{\mathbf{v}}
$$

where $x^{\mathbf{v}}=x_{1}^{v^{(1)}} x_{2}^{v^{(2)}} \cdots x_{d}^{v^{(d)}}$ is the monomial in $K\left[X_{1}, \ldots, X_{d}\right]$ associated to $\mathbf{v}=\left(v^{(1)}, v^{(2)}, \ldots, v^{(d)}\right)$. We consider the power series expansion of $1 /\left(1-F_{A}\right)$ the following formal series:

$$
P\left(F_{A}\right)=\sum_{k=0}^{\infty}\left(F_{A}\right)^{k}
$$

The following lemma ([5, Lemma 2.2] for $d=1)$ is obtained by applying Leibnitz's rule:

$$
\left(a_{1}+a_{2}+\cdots+a_{m}\right)^{n}=\sum_{h_{1}+h_{2}+\cdots+h_{m}=n} \frac{n !}{h_{1} ! h_{2} ! \ldots h_{m} !} a_{1}^{h_{1}} a_{2}^{h_{2}} \cdots a_{m}^{h_{m}} .
$$

Lemma 3.2. Let $A=\left\{\boldsymbol{a}_{1}, \boldsymbol{a}_{2}, \ldots, \boldsymbol{a}_{n}\right\} \subseteq \mathbb{N}^{d}$ and $\boldsymbol{b} \in \mathbb{N}^{d}$. Then $\boldsymbol{b}$ is a linear combination of $\boldsymbol{a}_{1}, \boldsymbol{a}_{2}, \ldots, \boldsymbol{a}_{n}$ with nonnegative integer coefficients if and only if the coefficient of $x^{b}$ in $P\left(F_{A}\right)$ is nonzero. 
Proof. By Leibnitz's rule we obtain:

$$
\begin{aligned}
& \left(F_{A}\right)^{t}=\left(x_{1}^{a_{1}^{(1)}} x_{2}^{a_{1}^{(2)}} \cdots x_{d}^{a_{1}^{(d)}}+x_{1}^{a_{2}^{(1)}} x_{2}^{a_{2}^{(2)}} \cdots x_{d}^{a_{2}^{(d)}}+\cdots+x_{1}^{a_{n}^{(1)}} x_{2}^{a_{n}^{(2)}} \cdots x_{d}^{a_{n}^{(d)}}\right)^{t}=
\end{aligned}
$$

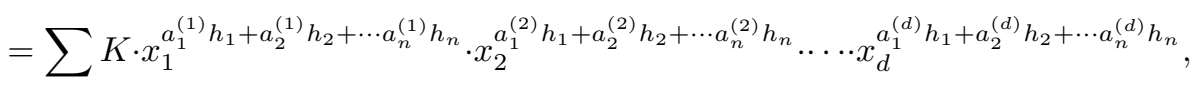

where the sum is extended to $h_{1}, \ldots, h_{n} \in \mathbb{N}$ with $h_{1}+\cdots+h_{n}=t$ and $K$ is a nonzero coefficient.

If $\mathbf{b}=\sum_{i=1}^{n} \lambda_{i} \mathbf{a}_{i}$, set $t=\sum_{i=1}^{n} \lambda_{i}$, then $x^{\mathbf{b}}$ is a monomial in $\left(F_{A}\right)^{t}$. Conversely, if $x^{\mathbf{b}}$ has nonzero coefficient in $P\left(F_{A}\right)$ then

$$
x^{\mathbf{b}}=x_{1}^{a_{1}^{(1)} h_{1}+a_{2}^{(1)} h_{2}+\cdots a_{n}^{(1)} h_{n}} \cdot x_{2}^{a_{1}^{(2)} h_{1}+a_{2}^{(2)} h_{2}+\cdots a_{n}^{(2)} h_{n}} \cdots \cdots x_{d}^{a_{1}^{(d)} h_{1}+a_{2}^{(d)} h_{2}+\cdots a_{n}^{(d)} h_{n}}
$$

with $h_{i} \in \mathbb{N}$ for $i=1, \ldots, n$ that is $\mathbf{b}=\sum_{i=1}^{n} h_{i} \mathbf{a}_{i}$.

Definition 3.3. Let $A=\left\{\mathbf{a}_{1}, \mathbf{a}_{2}, \ldots, \mathbf{a}_{n}\right\} \subseteq \mathbb{N}^{d}$ with $\mathbf{a}_{i}=\left(a_{i}^{(1)}, a_{i}^{(2)}, \ldots, a_{i}^{(d)}\right)$ for $i=1,2, \ldots, n$, and let $\mathbf{b} \in \mathbb{N}^{d}$.

Let $t=\min \left\{\sum_{j=1}^{d} a_{i}^{(j)} \mid i=1,2, \ldots, n\right\}$. We define the positive integer

$$
N_{\mathbf{b}}:=\left\lfloor\frac{\sum_{j=1}^{d} b^{(j)}}{t}\right\rfloor \text {. }
$$

Proposition 3.4. Let $A=\left\{\boldsymbol{a}_{1}, \boldsymbol{a}_{2}, \ldots, \boldsymbol{a}_{n}\right\} \subseteq \mathbb{N}^{d}$ and $\boldsymbol{b} \in \mathbb{N}^{d}$. Then $\boldsymbol{b} \in\langle A\rangle$ if and only if the coefficient of $x^{b}$ is nonzero in the polynomial:

$$
F\left(x_{1}, x_{2}, \ldots, x_{d}\right)=\sum_{k=0}^{N_{b}}\left(F_{A}\right)^{k} .
$$

Proof. By lemma 3.2 it is enough to show that the coefficient of $x^{\mathbf{b}}$ is zero in $F\left(x_{1}, \ldots, x_{d}\right)$ if and only if it is zero also in $P\left(F_{A}\right)$, that is $\sum_{k=0}^{\infty}\left(F_{A}\right)^{k}$.

We suppose that the coefficient of $x^{\mathbf{b}}$ is nonzero in $P\left(F_{A}\right)$. Then there exists $r \in \mathbb{N}$ such that $x^{\mathbf{b}}$ is a monomial in $\left(F_{A}\right)^{r}$. By Leibnitz's rule we obtain:

$$
\begin{aligned}
& \left(F_{A}\right)^{r}=\left(x_{1}^{a_{1}^{(1)}} x_{2}^{a_{1}^{(2)}} \cdots x_{d}^{a_{1}^{(d)}}+x_{1}^{a_{2}^{(1)}} x_{2}^{a_{2}^{(2)}} \cdots x_{d}^{a_{2}^{(d)}}+\cdots+x_{1}^{a_{n}^{(1)}} x_{2}^{a_{n}^{(2)}} \cdots x_{d}^{a_{n}^{(d)}}\right)^{r} \\
= & \sum_{\mathbf{h}} K \cdot x_{1}^{a_{1}^{(1)} h_{1}+a_{2}^{(1)} h_{2}+\cdots a_{n}^{(1)} h_{n}} \cdot x_{2}^{a_{1}^{(2)} h_{1}+a_{2}^{(2)} h_{2}+\cdots a_{n}^{(2)} h_{n} \cdots \cdots x_{d}^{a_{1}^{(d)}} h_{1}+a_{2}^{(d)} h_{2}+\cdots a_{n}^{(d)} h_{n}},
\end{aligned}
$$


where $\mathbf{h}=\left(h_{1}, \ldots, h_{n}\right)$ with $h_{1}+h_{2}+\cdots+h_{n}=r$ and $K$ is the correspondent coefficient, but we do not need its exact value.

If $x_{1}^{b^{(1)}} x_{2}^{b^{(2)}} \ldots x_{d}^{b^{(d)}}$ appears in the sum, then there exist $h_{1}, h_{2}, \ldots, h_{n}$ with $h_{1}+h_{2}+\cdots+h_{n}=r$, such that the following equalities are satisfied:

$$
\begin{gathered}
a_{1}^{(1)} h_{1}+a_{2}^{(1)} h_{2}+\cdots a_{n}^{(1)} h_{n}=b^{(1)} \\
a_{1}^{(2)} h_{1}+a_{2}^{(2)} h_{2}+\cdots a_{n}^{(2)} h_{n}=b^{(2)} \\
\vdots \\
a_{1}^{(d)} h_{1}+a_{2}^{(d)} h_{2}+\cdots a_{n}^{(d)} h_{n}=b^{(d)} .
\end{gathered}
$$

We sum the righ-hand side and the left-hand side of all equalities, obtaining that:

$$
\begin{aligned}
r & =h_{1}+h_{2}+\cdots+h_{n} \leq \\
& \leq\left(a_{1}^{(1)}+a_{1}^{(2)}+\cdots+a_{1}^{(d)}\right) h_{1}+\left(a_{2}^{(1)}+a_{2}^{(2)}+\cdots+a_{2}^{(d)}\right) h_{2}+\cdots+ \\
& +\left(a_{n}^{(1)}+a_{n}^{(2)}+\cdots+a_{n}^{(d)}\right) h_{n}=b^{(1)}+b^{(2)}+\cdots+b^{(d)} .
\end{aligned}
$$

Eventually, if $t=\min \left\{\sum_{j=1}^{d} a_{i}^{(j)} \mid i=1,2, \ldots, n\right\}$ then $\frac{\sum_{j=1}^{d} a_{i}^{(j)}}{t} \geq 1$ for $i=1,2, \ldots, d$. So we can divide the right-hand side of inequality by $t$ and we obtain:

$$
\begin{aligned}
r & =h_{1}+h_{2}+\cdots+h_{n} \leq \\
& \leq \frac{\sum_{j=1}^{d} a_{1}^{(j)}}{t} h_{1}+\frac{\sum_{j=1}^{d} a_{2}^{(j)}}{t} h_{2}+\cdots+\frac{\sum_{j=1}^{d} a_{n}^{(j)}}{t} h_{n}=\frac{b^{(1)}+b^{(2)}+\cdots+b^{(d)}}{t}
\end{aligned}
$$

It follows that $r \leq N_{\mathbf{b}}$. So, if the coefficient of $x^{\mathbf{b}}$ in $P\left(F_{A}\right)$ is nonzero then the greatest power in which it is obtained is at last $N_{\mathbf{b}}$, for greater powers we are sure that monomial does not appear.

An application of the previous proposition is the following criterion for the existence of $\mathbb{N}$-solutions in a linear system with nonnegative integer coefficients.

Corollary 3.5. Let $M$ be a $d \times n$ matrix with entries in $\mathbb{N}$ whose columns are the vectors of the set $A=\left\{\boldsymbol{a}_{1}, \boldsymbol{a}_{2}, \ldots, \boldsymbol{a}_{n}\right\}$ and let $\boldsymbol{b} \in \mathbb{N}^{d}$. Then the linear system $M \boldsymbol{x}=\boldsymbol{b}$ admits solutions $\boldsymbol{x} \in \mathbb{N}^{n}$ if and only if the coefficient of $x^{\boldsymbol{b}}$ is nonzero in the polynomial:

$$
F\left(x_{1}, x_{2}, \ldots, x_{d}\right)=\sum_{k=0}^{N_{b}}\left(F_{A}\right)^{k} .
$$


The previous arguments suggest the following results.

Corollary 3.6. Let $S \subseteq \mathbb{N}^{d}$ be a $G N S, A=\left\{\boldsymbol{a}_{1}, \boldsymbol{a}_{2}, \ldots, \boldsymbol{a}_{n}\right\}$ be a finite system of generators for $S$ and $\boldsymbol{v} \in \mathbb{N}^{d}$. Then $\boldsymbol{v} \in S$ if and only if the coefficient of $x^{v}$ is nonzero in the polynomial:

$$
F\left(x_{1}, x_{2}, \ldots, x_{d}\right)=\sum_{k=0}^{N_{v}}\left(F_{A}\right)^{k} .
$$

If $S$ is a GNS and a finite system of generators for $S$ is known, then Corollary 3.6 provides a way to establish whether an element $\mathbf{v} \in S$. Furthermore it can be done with a finite computation, that is the building of a polynomial.

Remark 3.7. Recall that if $S \subseteq \mathbb{N}^{d}$ is a GNS and $A$ a finite system of generators for $S$, by Theorem $2.8 \mathrm{~A}$ satisfies the following conditions:

1. For all $j=1,2, \ldots, d$, there exist $a_{1}^{(j)} \mathbf{e}_{j}, a_{2}^{(j)} \mathbf{e}_{j}, \ldots, a_{n_{j}}^{(j)} \mathbf{e}_{j} \in A$ such that $G C D\left(a_{1}^{(j)}, a_{2}^{(j)}, \ldots, a_{n_{j}}^{(j)}\right)=1$

2. For every $i, k \in\{1,2, \ldots, d\}$ with $i<k$ there exist $\mathbf{x}, \mathbf{y} \in A$ such that $\mathbf{x}=\mathbf{e}_{i}+n_{i}^{(k)} \mathbf{e}_{k}$ and $\mathbf{y}=\mathbf{e}_{k}+n_{k}^{(i)} \mathbf{e}_{i}$ with $n_{i}^{(k)}, n_{k}^{(i)} \in \mathbb{N}$.

For every $j=1,2, \ldots, d$, let $S_{j}$ be the numerical semigroup generated by $\left\{a_{1}^{(j)}, a_{2}^{(j)}, \ldots, a_{n_{j}}^{(j)}\right\}$. We denote by $F^{(j)}$ the Frobenius number of $S_{j}$. Let $\mathbf{v}=\left(v^{(1)}, v^{(2)}, \ldots, v^{(d)}\right) \in \mathbb{N}^{d}$ be the element defined by

$$
v^{(j)}=\sum_{i \neq j}^{d} F^{(i)} n_{i}^{(j)}+F^{(j)} .
$$

It is proved that $H(S) \subseteq \pi(\mathbf{v})$ (Corollary 2.9), and $\pi(\mathbf{v})$ is a finite set.

We conclude giving a simple algorithm to compute the set of holes of $S$, that is $H(S)$, if a finite system of generators for $S$ is known.

\section{Algorithm.}

Let $S \subseteq \mathbb{N}^{d}$ be a GNS and $A=\left\{\mathbf{a}_{1}, \mathbf{a}_{2}, \ldots, \mathbf{a}_{n}\right\}$ be a finite system of generators of $S$. To compute $H(S)$ we have to do the following steps:

1. Compute the element $\mathbf{v}$ of the Remark 3.7.

2. For all $\mathbf{x} \in \pi(\mathbf{v})$ we verify: if $\mathbf{x}$ is not a $\mathbb{N}$-linear combination of elements in $A$ then $\mathrm{x} \in H(S)$. This check can be done by Corollary 3.6.

At the end of the second step the set $H(S)$ is computed. 


\section{References}

[1] Bras-Amorós, M.: Fibonacci-like behavior of the number of numerical semigroups of a given genus. Semigroup Forum 76, 379-384 (2008).

[2] Bras-Amorós, M.: Bounds on the number of numerical semigroups of a given genus. J. Pure Appl.Algebra 213, 997-1001 (2009).

[3] Failla, G., Peterson, C., Utano, R.: Algorithms and basic asymptotics for generalized numerical semigroups in $\mathbb{N}^{d}$, Semigroup Forum 92(2), 460473 (2016).

[4] Fröberg, R., Gottlieb, C., Häggkvist, R.: On numerical semigroups. Semigroup Forum 35, 63-83 (1986/1987).

[5] Martino, I., Martino, L.: On the variety of linear recurrences and numerical semigroups. Semigroup Forum 88, 569-574 (2014).

[6] Pisón-Casares, P., Vigneron-Tenorio, A.: $\mathbb{N}$-solutions to linear systems over $\mathbb{Z}$. Linear Algebra Its Appl. 384, 135-154 (2004).

[7] Rosales, J.C.: On finitely generated submonoids of $\mathbb{N}^{k}$. Semigroup Forum 50, 251-262 (1995).

[8] J. C. Rosales, P. A. García-Sánchez, On Cohen-Macaulay and Gorenstein simplicial affine semigroups, Proc. Edinburgh Math. Soc. 41 (1998), 517537.

[9] Rosales, J.C., García-Sánchez, P.A.: Numerical Semigroups, Developments in Mathematics, vol. 20. Springer, New York (2009).

[10] Rosales, J.C., García-Sánchez, P.A., García-García, J.I., Jiménez Madrid, J.A.: The oversemigroups of a numerical semigroup, Semigroup Forum 67(1), 145-158, (2003).

Carmelo CISTO

Universitá di Messina,

Dipartimento di Scienze Matematiche e Informatiche, Scienze Fisiche e

Scienze della Terra,

Viale Ferdinando Stagno D'Alcontres 31,

98166 Messina, Italy

Email: carmelo.cisto@unime.it

Gioia FAILLA,

Universitá Mediterranea di Reggio Calabria,

Dipatimento DIIES,

Via Graziella, Feo di Vito,

Reggio Calabria, Italy.

Email: gioia.failla@unirc.it

Rosanna UTANO

Universitá di Messina,

Dipartimento di Scienze Matematiche e Informatiche, Scienze Fisiche e

Scienze della Terra,

Viale Ferdinando Stagno D'Alcontres 31,

98166 Messina, Italy

Email: rosanna.utano@unime.it 